\title{
Cisplatin Versus Carboplatin and Paclitaxel in Radiochemotherapy for Patients With Locally Advanced Head and Neck Squamous Cell Carcinoma
}

\author{
SANDY NASSIF ${ }^{1}$, JORN WICHMANN ${ }^{1}$, DOMINIC STRUBE ${ }^{2}$, STRATOS VASSIS $^{1}$, \\ HANS CHRISTIANSEN $^{1}$ and DIANA STEINMANN ${ }^{1}$ \\ ${ }^{1}$ Clinic for Radiotherapy and Special Oncology of the Hannover Medical School, Hannover, Germany; \\ ${ }^{2}$ University of Applied Sciences, Technology, Business and Design Wismar, Wismar, Germany
}

\begin{abstract}
Background/Aim: The implementation of a platinum-containing regimen is recommended for definitive and adjuvant therapy of patients with locally advanced head and neck tumour. We compared the conditions for the use of cisplatin or carboplatin/paclitaxel or for changing between these two regimens on a clinic-specific basis. Patients and Methods: We evaluated 150 patients with advanced head and neck squamous cell carcinoma who received simultaneous radiochemotherapy at our institution between 2012 and 2017. Chemotherapy with weekly doses of cisplatin (40 $\mathrm{mg} / \mathrm{m}^{2}$, group 1) or, in cases of impaired renal and/or cardiac function, with weekly doses of carboplatin AUC2 and paclitaxel (45 mg/m ${ }^{2}$, group 2), was performed as a first-choice therapy. If toxicities occurred in group 1 , treatment was switched to the carboplatin/paclitaxel regimen (group 3). Patient- and therapy-related parameters, toxicity and survival data were compared across groups. Results: We examined 99, 30, and 21 patients in each group who received at least 1 course of chemotherapy. Group 3 patients switched from cisplatin to carboplatin/paclitaxel after a median of 3 courses due to nephrotoxicity (95.2\%). The target of at least 5 chemotherapy courses was most frequently achieved by patients in group 1 (69.7\%), followed by group 3 (61.9\%) and then group $2(40.0 \%)$. Multivariate analysis revealed that patients who switched groups were more likely to be over 60 years old $(p=0.021)$, undergo definitive
\end{abstract}

This article is freely accessible online.

Correspondence to: Sandy Nassif, Clinic for Radiotherapy and Special Oncology of the Hannover Medical School, Carl-NeubergStraße 1, 30625 Hannover, Germany. Tel: +49 5115322574, e-mail: nassif.sandy@mh-hannover.de

Key Words: Charlson comorbidity index, definitive therapy, haematotoxicity, nephrotoxicity, regimen change. radiochemotherapy $(p=0.049)$ and develop higher nephrotoxicity $(p=0.036)$ than group 1 patients. Outcomes did not differ between groups. Conclusion: When cisplatin application is contraindicated due to renal- or cardiotoxicity, carboplatin/paclitaxel is an appropriate option.

In definitive and adjuvant radiochemotherapy (RCT) of locally advanced head and neck tumour patients, different chemotherapy (CTx) regimens are applied depending on specific clinical criteria. The guideline recommendation foresees the utilization of a platinum-containing regimen ( 1 , 2 ), with cisplatin being the most commonly advocated and used at a cumulative dose of at least $200 \mathrm{mg} / \mathrm{m}^{2}(3,4)$. At our institution, we prefer weekly administration of cisplatin 40 $\mathrm{mg} / \mathrm{m}^{2}$ body surface area (BSA) (5). We observed an increased incidence of renal toxicity following administration of cisplatin, as did other studies $(6,7)$. Some patients have initially high renal parameters or cardiac comorbidities, disqualifying them for cisplatin use. In these circumstances, a carboplatin-based regimen is administered $(8,9)$. Carboplatin, with its radiosensitizing properties and lower renal effect than cisplatin, qualifies under National Comprehensive Cancer Network (NCCN) guidelines as an effective alternative for patients for whom cisplatin is not appropriate. Phase II trials of carboplatin-oriented concurrent RCTs have shown complete response rates of $65.0 \%-70.0 \%$, similar to those seen with cisplatin. Noronha et al. concluded that a carboplatin-based RCT is well tolerated by patients who are ineligible for cisplatin and appears to provide superior outcomes than those reported in trials of radiotherapy (RT) alone (10). A combination of carboplatin and paclitaxel has previously been described in the context of head and neck tumours (11-13). In addition, carboplatin acts as a radiosensitizer in other entities, often in conjunction with paclitaxel, as exemplified in the setting of CTx for oesophageal $(14,15)$ and lung cancer $(16)$. In many cases, initial therapy with cisplatin is accompanied by the occurrence 
of renal toxicity, so changing the cytostatic agent to carboplatin allows for continued chemotherapeutic treatment.

The overall objective of this study was to compare the conditions for using cisplatin or carboplatin/paclitaxel or for changing between the two regimens in a clinic-specific manner. Therefore, we analysed the survival parameters of the two cytostatic regimens, cisplatin vs. carboplatin/paclitaxel, and their application criteria. Finally, we examined whether a direct correlation exists between renal toxicity or the Charlson Comorbidity Index (CCI) and cytostatic regimen change.

\section{Patients and Methods}

Patients and treatment. For our retrospective observational study comparing cisplatin and carboplatin/paclitaxel, data were collected from patients who underwent simultaneous RCT as part of the multimodal treatment of advanced head and neck squamous cell carcinoma at our institution between May 2012 and December 2017. Patient-related demographical and treatment data were gathered from records and analysed for each group separately as well as for all patients as a whole group. The procedure was performed according to the guidelines of the Ethics Committee and the Data Protection Department of the same institution (Ethics Application Number: 9250_BO_K_2020).

In the case of pre-RCT surgery, resection status ( $\mathrm{R} \mathrm{X} / 0$ vs. $\mathrm{R} 1 / 2)$ and time interval between surgery and RT ( $\leq 6$ weeks $v s .>6$ weeks) were recorded. By default, our RT concept involves the use of intensity-modulated radiation therapy (IMRT) or volumetric modulated arc therapy (VMAT) with simultaneous integrated boost (SIB) as image-guided radiotherapy (IGRT). In the frame of definitive therapy, doses of 66 (2.2) Gy to the primary tumour, 60 (2.0) Gy to pathologic lymph nodes, and 54 (1.8) Gy to the corresponding lymph node area are common, with the first number representing the total dose and the number in parentheses representing the single dose. However, in adjuvant therapy, 62.4 (2.08) Gy to the tumour region and 54 (1.8) Gy to the corresponding lymph node area are typically applied. Adjuvant radiochemotherapy was indicated for R1 resection or extracapsular extension (ECE), otherwise for large tumours (T4, N3) and optionally at the patient's request. In case of lymph node involvement without ECE, adjuvant radiotherapy represented the treatment of choice. For parallel group comparisons, our patients were divided into 3 groups as follows: patients in group 1 were treated exclusively with cisplatin, with a weekly administration of $40 \mathrm{mg} / \mathrm{m}^{2} \mathrm{BSA}$; in group 2, carboplatin AUC2+paclitaxel (45 mg/m² BSA) was initially preferred for treatment due to comorbidities. Group 3 consisted of patients who started with cisplatin and switched to carboplatin/paclitaxel during the course of therapy because of toxicity. We aimed for at least 5 (adjuvant treatment) to 6 (definitive treatment) CTx courses for optimal outcomes. To calculate whether or to what extent the target total dose was achieved, we assumed 5 as the intended number of courses. Only patients with squamous cell carcinoma of the head and neck were included; prior CTx or RT to the head and neck area were exclusion criteria.

CCI was determined with and without taking age into account In the first step, points are summed for each patient according to their comorbidities (e.g., heart attack, myocardial infarction), with a small score corresponding to a low comorbidity burden and vice versa (17-20). In addition, points are assigned to different age ranges and can be added to the comorbidity score, thus forming the Age-Adjusted Charlson Comorbidity Index (ACCI) (21-23). The age span of our patients was 33 to 86 years. Panendoscopy, computer tomography of the head and neck as well as bone scan, chest X-ray and ultrasound of the neck nodes and abdomen were all parts of the staging process $(24,25)$. Tumour stage was determined with the aid of the TNM Classification of Malignant Tumours according to the Union Internationale Contre Le Cancer (UICC) (26). If the date of the initial diagnosis was before January 3, 2017, the 7th edition was utilized; thereafter, the 8th edition was applied. Tumours occupying more than one location were categorized as multilevel tumours.

Toxicity. For nephrotoxicity and haematotoxicity, the nadir values of parameters such as creatinine, leukocytes, haemoglobin and platelets were noted. For toxicity classification, we deliberately chose to apply CTCAE versions 3 and 5, as each categorizes nephrotoxicity and haematotoxicity in its own way consistent with our needs. For haematotoxicity, CTCAE version 3 (27) was followed with separate determinations of leukocytes, platelets and haemoglobin to identify the main cause of haematotoxicity; subsequently, the highest of these three detected grades was adopted as the overall haematotoxicity grade. For one analysis, the occurrence and non-occurrence of haematotoxicity was distinguished (grade 0 vs. grade 1-4), and for the other, differentiation was made between grade $1 / 2$ and grade $3 / 4$. To determine the degree of renal toxicity, CTCAE version 5 was consulted, more precisely the sub-item "Investigations." For creatinine levels, toxicity grade assignment was guided by the following normal value limits: for men 59-104 $\mu \mathrm{mol} / 1$, for women $45-48 \mu \mathrm{mol} / \mathrm{l}$. Considering male patients, grade 1 corresponded to a creatinine value between 105 and $156 \mu \mathrm{mol} / 1$, whereas grade 2 corresponded to a creatinine value between 157 and $312 \mu \mathrm{mol} / \mathrm{l}$; however, in women, grade 1 was defined by a creatinine value between 85 and $126 \mu \mathrm{mol} / 1$, while grade 2 was between 127 and $252 \mu \mathrm{mol} / 1$. Assessment of renal toxicity distinguished between grade 0 (normal values) and grades 1 or 2 .

Ethics approval. This study is compliant with the ethical standards of the committees on human experimentation (institutional and national) and with the Declaration of Helsinki. It was carried out according to the guidelines of the Ethics Committee and the Data Protection Department of the Hannover Medical School (Ethics Application Number: 9250_BO_K_2020). All patients gave their informed consent before participating in the study.

Statistical analysis. Statistical analysis was performed using IBM SPSS Statistics Version 27. The date of the first therapy day and the last contact or death served as references for calculating follow-up time. Due to small sample sizes, the two-tailed Fisher's exact test (28) was applied to compare patient characteristics plus RCT and toxicity data between groups 1 and 2 and groups 1 and 3 . The null hypothesis is that no relationship exists between expected and observed frequencies. The influence of individual factors on the change in cytostatic agents was investigated by means of univariate logistic regression, in which odds ratios were given with a confidence interval of $95 \%$. The independent variables were then compared using multivariate logistic regression. During this process, all parameters not showing a significant correlation with cytostatic 
drug change were successively removed from the analysis through backward elimination beginning with the highest $p$-value. To compare groups 1 and 2 for 3-year survival, binary logistic regression was utilized. Finally, the two-tailed Fisher's exact test was also applied for recurrence and metastasis rates as well as for toxicity comparing each group with the other two. Survival rates were depicted using Kaplan-Meier curves. Overall survival (OS), progression-free survival (PFS) and locoregional control (LRC) were investigated in the different therapy groups, with two of the three groups examined per analysis run. Due to the study's retrospective nature, no distinction could be drawn between tumourand nontumour-related deaths.

\section{Results}

Patient characteristics. As shown in Table I, we enrolled a total of 150 patients who received at least 1 course of CTx in group $1 / 2 / 3$ with $n=99 / 30 / 21$. ACCI scores were higher in groups 2 and 3 than in group 1: almost half of the cisplatin patients, $49(49.5 \%)$, scored $<4$, whereas more patients in groups 2 and 3 achieved an ACCI score $\geq 4$, namely, 26 of the carboplatin/paclitaxel $(86.7 \%)$ and 17 of the regimen change patients $(81.0 \%)$.

Number of chemotherapy courses and reasons for change or cancellation. The target of at least 5 courses of CTx was achieved most often in group $1(69.7 \%)$ but only by $40.0 \%$ in group 2 and $61.9 \%$ in the regimen change group. Two of the 99 cisplatin $(2.0 \%)$ and one of the 30 group 2 patients (3.3\%) received only one CTx course - in the latter case because of paclitaxel allergy and in the other two cases at patient request. Overall, 20 patients underwent dose reduction, most of whom were cisplatin patients (Table II). In the majority of cases, the dose was reduced from the $5^{\text {th }}$ course onward due to deteriorated blood values, with $50.0 \%$ being the most frequent reduction. Ninety-five percent of patients in group 3 switched regimens as a result of worsened renal parameters.

In univariate analysis, we compared group 1 patients who were continuously treated with cisplatin and group 3 patients who started therapy with cisplatin and subsequently changed to carboplatin/paclitaxel. Such switching, as observed in group 3, was associated with an age of over $60(p=0.006)$, an ACCI $\geq 4(p=0.016)$, definitive therapy $(p=0.020)$ and nephrotoxicity level $1 / 2(p=0.001)$. Multivariate analysis revealed that patients who changed the cytostatic drug were more likely to be over 60 years old $(p=0.021)$, undergo definitive therapy $(p=0.049)$ and develop a nephrotoxicity grade $1 / 2(p=0.036)$ than patients treated with cisplatin only when all other variables were held constant. Elevating the significance level from $p<0.050$ to $p<0.100$ produced the same significance. The results of univariate and multivariate analysis for the comparison of the cisplatin and change groups are summarized in Table III.
Toxicity. Patients treated exclusively with cisplatin (Group 1) were comparatively least likely to reach CTCAE haematotoxicity grade 3/4, with 19 patients (19.2\%). In Group 2 and Group 314 patients $(46.7 \%)$ and 8 patients (38.1\%), respectively, developed haematotoxicity grade $3 / 4$. Regarding nephrotoxicity, group 1 had two patients $(2.0 \%)$ that achieved a CTCAE grade 2 toxicity level in the nadir compared with one patient in group $2(3.3 \%)$ and two patients in group $3(9.5 \%)$. Finally, none of the patients showed nephrotoxicity levels of $3 / 4$.

Fisher's exact tests showed a difference in the occurrence of nephrotoxicity grade $1 / 2(p=0.011)$, haematotoxicity grade $1-4$ ( $p=0.015)$ or grade $3 / 4(p=0.003)$ between the cisplatin arm and the other arms. Furthermore, a dependence was observed between group 2 and the occurrence of grade $3 / 4$ haematotoxicity $(p=0.012)$. Compared to groups 1 and 2 , the regimen change group displayed a dependence on the incidence of nephrotoxicity grade $1 / 2(p=0.003)$. All results of Fisher's exact tests are summarized in Table IV. Beyond switching to carboplatin/paclitaxel, cisplatin-induced toxicity did not lead to relevantly stronger pronounced treatment discontinuation or dose reduction in group 1 patients compared to the other two groups, yet the carboplatin/paclitaxel-only patients accomplished fewer chemotherapy courses compared with the cisplatin-only patients. The change group contained too few patients for statistical analysis, but the majority completed 5 courses or more, as shown in Table II.

Survival. Median follow-up was 41 months for patients in all groups, specifically, 42 in group 1, 41 in group 2 and 36 in group 3. During the follow-up period, a total of 47 patients died, of whom 17 experienced progression of their cancer after therapy termination. No significant difference in OS, PFS or LRC was found among the groups (Figure 1, Figure 2, and Figure 3). Also, a separate evaluation of patients with definitive or adjuvant therapy did not reveal any significant differences in terms of OS, PFS, and LRC when comparing the different groups with each other.

The Kaplan-Meier evaluations demonstrated 3-year OS rates of $73.3 \%$ in the cisplatin Group 1, 56.3\% in Group 2 and $77.4 \%$ in the Change Group. Additionally, 3-year PFS rates of $72.6 \%$ in cisplatin patients, $68.9 \%$ in group 2 and $81.6 \%$ in regimen change patients were observed. The 3 -year LRC rates were $83.1 \%$ in the cisplatin group compared to $88.3 \%$ in Group 2 and $88.7 \%$ in the Change Group. The comparison of 3 -year survival between the cisplatin and carboplatin/paclitaxel groups in multivariate logistic regression showed no significant difference.

\section{Discussion}

This retrospective study compared the preconditions and criteria for using cisplatin or carboplatin/paclitaxel or 
Table I. Patient characteristics.

\begin{tabular}{|c|c|c|c|c|c|c|}
\hline \multirow[t]{2}{*}{ Categories } & \multirow[t]{2}{*}{$\begin{array}{c}\text { Total } \\
\text { Number }\end{array}$} & \multirow{2}{*}{$\begin{array}{c}\text { Cisplatin } \\
\text { Group } 1 \\
\text { n }(\%)\end{array}$} & \multirow{2}{*}{$\begin{array}{c}\text { Carboplatin/ } \\
\text { Paclitaxel } \\
\text { Group } 2 \\
\text { n }(\%)\end{array}$} & \multirow{2}{*}{$\begin{array}{c}\text { Regimen } \\
\text { Change } \\
\text { Group } 3 \\
\text { n (\%) }\end{array}$} & \multicolumn{2}{|c|}{$\begin{array}{c}\text { Fisher's Exact } \\
p \text {-Value }\end{array}$} \\
\hline & & & & & $\begin{array}{l}\text { Group } 1 \text { vs. } \\
\text { Group } 2\end{array}$ & $\begin{array}{c}\text { Group } 1 \text { vs } \\
\text { Group } 3\end{array}$ \\
\hline Number of patients & $\mathrm{n}=150$ & 99 & 30 & 21 & \multirow{2}{*}{\multicolumn{2}{|c|}{$\leq 60 \mathrm{vs} .>60$}} \\
\hline \multicolumn{5}{|l|}{ Age (years) } & & \\
\hline$\leq 60$ & $\mathrm{n}=70$ & $58(58.6)$ & $7(23.3)$ & $5(23.8)$ & \multirow[t]{2}{*}{0.001} & 0.007 \\
\hline$>60$ & $\mathrm{n}=80$ & $41(41.4)$ & $23(76.7)$ & $16(76.2)$ & & \\
\hline \multicolumn{5}{|l|}{ Gender } & \multicolumn{2}{|c|}{ Female $v s$. Male } \\
\hline Female & $\mathrm{n}=39$ & $27(27.3)$ & $8(26.7)$ & $4(19.0)$ & \multirow[t]{2}{*}{1.000} & 0.586 \\
\hline Male & $\mathrm{n}=111$ & $72(72.7)$ & $22(73.3)$ & $17(81.0)$ & & \\
\hline \multicolumn{5}{|l|}{ Tumour category } & \multicolumn{2}{|c|}{ Т $0 / 1 / 2 v s . \mathrm{T} 3 / 4$} \\
\hline Т 0 & $\mathrm{n}=13$ & $9(9.2)$ & $2(6.7)$ & $2(9.5)$ & 0.010 & 0.327 \\
\hline $\mathrm{T} 1 / 2$ & $\mathrm{n}=40$ & $33(33.7)$ & $3(10.0)$ & $4(19.0)$ & & \\
\hline Т $3 / 4$ & $\mathrm{n}=96$ & $56(57.1)$ & $25(83.3)$ & $15(71.4)$ & & \\
\hline \multicolumn{5}{|l|}{ Lymph nodes category } & \multicolumn{2}{|c|}{$\mathrm{N} 0 / 1$ vs. $\mathrm{N} 2 / 3$} \\
\hline $\mathrm{N} 0 / 1$ & $\mathrm{n}=45$ & $33(33.7)$ & $7(23.3)$ & $5(23.8)$ & 0.370 & 0.448 \\
\hline $\mathrm{N} 2 / 3$ & $\mathrm{n}=104$ & $65(66.3)$ & $23(76.7)$ & $16(76.2)$ & & \\
\hline \multicolumn{7}{|l|}{ Metastases category } \\
\hline M 0 & $\mathrm{n}=147$ & $98(100.0)$ & $29(96.7)$ & $20(95.2)$ & & \\
\hline M 1 & $\mathrm{n}=2 *$ & $0(0.0)$ & $1(3.3)$ & $1(4.8)$ & & \\
\hline \multicolumn{5}{|l|}{ UICC stage } & \multicolumn{2}{|c|}{ Stage III vs. Stage IV } \\
\hline Stage I/II & $\mathrm{n}=3$ & $2(2.0)$ & $0(0.0)$ & $1(4.8)$ & 1.000 & 0.193 \\
\hline Stage III & $\mathrm{n}=23$ & $17(17.3)$ & $5(16.7)$ & $1(4.8)$ & & \\
\hline Stage IV & $\mathrm{n}=123$ & $79(80.6)$ & $25(83.3)$ & $19(90.5)$ & & \\
\hline \multicolumn{5}{|l|}{ Tumour site } & Multile & tumour \\
\hline Lip, oral cavity & $\mathrm{n}=34$ & $28(28.3)$ & $3(10.0)$ & $3(14.3)$ & Yes & No \\
\hline Oropharynx & $\mathrm{n}=40$ & $27(27.3)$ & $9(30.0)$ & $4(19.0)$ & 0.240 & 0.762 \\
\hline Hypopharynx & $\mathrm{n}=18$ & $6(6.1)$ & $8(26.7)$ & $4(19.0)$ & & \\
\hline Nasopharynx & $\mathrm{n}=3$ & $1(1.0)$ & $0(0.0)$ & $2(9.5)$ & & \\
\hline Larynx & $\mathrm{n}=18$ & $11(11.1)$ & $5(16.7)$ & $2(9.5)$ & & \\
\hline Nose, paranasal sinuses & $\mathrm{n}=1$ & $1(1.0)$ & $0(0.0)$ & $0(0.0)$ & & \\
\hline Large salivary glands & $\mathrm{n}=3$ & $2(2.0)$ & $1(3.3)$ & $0(0.0)$ & & \\
\hline CUP, lymph node metastasis & $\mathrm{n}=10$ & $6(6.1)$ & $2(6.7)$ & $2(9.5)$ & & \\
\hline Multilevel tumour & $\mathrm{n}=23$ & $17(17.2)$ & $2(6.7)$ & $4(19.0)$ & & \\
\hline Treated tumour is a recurrence & & & & & Yes & No \\
\hline Yes & $\mathrm{n}=23$ & $18(18.2)$ & $4(13.3)$ & $1(4.8)$ & 0.782 & 0.190 \\
\hline No & $\mathrm{n}=127$ & $81(81.8)$ & $26(86.7)$ & $20(95.2)$ & & \\
\hline CCI & & & & & & \\
\hline 2 & $\mathrm{n}=104$ & $76(76.8)$ & $13(43.3)$ & $15(71.4)$ & & \\
\hline 3 & $\mathrm{n}=22$ & $15(15.2)$ & $5(16.7)$ & $2(9.5)$ & & \\
\hline 4 & $\mathrm{n}=16$ & $6(6.1)$ & $7(23.3)$ & $3(14.3)$ & & \\
\hline$\geq 5$ & $\mathrm{n}=8$ & $2(2.0)$ & $5(16.7)$ & $1(4.8)$ & & \\
\hline ACCI & & & & & & \\
\hline 2 & $\mathrm{n}=17$ & $16(16.2)$ & $0(0.0)$ & $1(4.8)$ & & \\
\hline 3 & $\mathrm{n}=40$ & $33(33.3)$ & $4(13.3)$ & $3(14.3)$ & & \\
\hline 4 & $\mathrm{n}=43$ & $25(25.3)$ & $6(20.0)$ & $12(57.1)$ & & \\
\hline 5 & $\mathrm{n}=22$ & $16(16.2)$ & $5(16.7)$ & $1(4.8)$ & & \\
\hline 6 & $\mathrm{n}=15$ & $7(7.1)$ & $6(20.0)$ & $2(9.5)$ & & \\
\hline$\geq 7$ & $\mathrm{n}=13$ & $2(2.0)$ & $9(30.0)$ & $2(9.5)$ & & \\
\hline ACCI, Dichotomized & & & & & $<4$ & $\geq 4$ \\
\hline$<4$ & $\mathrm{n}=57$ & $49(49.5)$ & $4(13.3)$ & $4(19.0)$ & 0.000 & 0.015 \\
\hline$\geq 4$ & $\mathrm{n}=93$ & $50(50.5)$ & $26(86.7)$ & $17(81.0)$ & & \\
\hline Follow-up median [Range (months)] & $41(1-97)$ & $42(1-97)$ & $41(4-74)$ & $36(5-81)$ & & \\
\hline
\end{tabular}

*Solitary pulmonary metastasis, questionable or excision performed. CCI: Charlson Comorbidity Index; ACCI: age-adjusted Charlson Comorbidity Index; UICC: Union Internationale Contre le Cancer. Bold $p$-Values indicate statistical significance. 
Table II. Radiochemotherapy characteristics and toxicities.

\begin{tabular}{|c|c|c|c|c|c|c|}
\hline \multirow[t]{2}{*}{ Categories } & \multirow[t]{2}{*}{$\begin{array}{c}\text { Total } \\
\text { Number }\end{array}$} & \multirow{2}{*}{$\begin{array}{l}\text { Cisplatin } \\
\text { Group } 1 \\
\mathrm{n}(\%)\end{array}$} & \multirow{2}{*}{$\begin{array}{c}\text { Carboplatin/ } \\
\text { Paclitaxel } \\
\text { Group } 2 \\
\text { n }(\%)\end{array}$} & \multirow{2}{*}{$\begin{array}{l}\text { Regimen } \\
\text { Change } \\
\text { Group } 3 \\
\text { n }(\%)\end{array}$} & \multicolumn{2}{|c|}{$\begin{array}{c}\text { Fisher's Exact } \\
p \text {-Value }\end{array}$} \\
\hline & & & & & $\begin{array}{l}\text { Group } 1 \text { vs. } \\
\text { Group } 2\end{array}$ & $\begin{array}{l}\text { Group } 1 v s . \\
\text { Group } 3\end{array}$ \\
\hline \multicolumn{5}{|l|}{ Therapy category } & \multicolumn{2}{|c|}{ Definitive $v s$. Adjuvant } \\
\hline Definitive & $\mathrm{n}=79$ & $42(42.4)$ & $22(73.3)$ & $15(71.4)$ & 0.004 & 0.018 \\
\hline Adjuvant & $\mathrm{n}=71$ & $57(57.6)$ & $8(26.7)$ & $6(28.6)$ & \multirow{2}{*}{\multicolumn{2}{|c|}{$\mathrm{R} X / 0$ vs. R $1 / 2$}} \\
\hline \multicolumn{5}{|l|}{ Extent of resection } & & \\
\hline R X/0 & $\mathrm{n}=51$ & $41(78.8)$ & $6(75.0)$ & $4(66.7)$ & \multirow[t]{2}{*}{1.000} & 0.608 \\
\hline $\mathrm{R} 1 / 2$ & $\mathrm{n}=15$ & $11(21.2)$ & $2(25.0)$ & $2(33.3)$ & & \\
\hline \multicolumn{5}{|l|}{ Interval surgery to radiotherapy } & \multicolumn{2}{|c|}{$\leq 6$ Weeks $v s .>6$ Weeks } \\
\hline$\leq 6$ Weeks & $\mathrm{n}=11$ & $7(12.3)$ & $1(12.5)$ & $3(50.0)$ & 1.000 & 0.048 \\
\hline$>6$ Weeks & $\mathrm{n}=60$ & $50(87.7)$ & $7(87.5)$ & $3(50.0)$ & & \\
\hline \multicolumn{5}{|l|}{ Neck dissection before radiotherapy } & Yes & No \\
\hline Yes & $\mathrm{n}=66$ & $51(51.5)$ & $9(30.0)$ & $6(28.6)$ & 0.059 & 0.091 \\
\hline No & $\mathrm{n}=84$ & $48(48.5)$ & $21(70.0)$ & $15(71.4)$ & & \\
\hline Boost modality & & & & & & \\
\hline SIB & $\mathrm{n}=141$ & $93(94.9)$ & $28(100.0)$ & $20(100.0)$ & & \\
\hline Sequential boost & $\mathrm{n}=2$ & $2(2.0)$ & $0(0.0)$ & $0(0.0)$ & & \\
\hline SIB and sequential Boost & $\mathrm{n}=3$ & $3(3.1)$ & $0(0.0)$ & $0(0.0)$ & & \\
\hline RTT median [Range (days)] & $43(9-73)$ & $42(9-73)$ & $43(36-53)$ & $43(39-50)$ & & \\
\hline RTT >49 Days & & & & & Yes & No \\
\hline Yes & $\mathrm{n}=11$ & $6(6.1)$ & $4(13.3)$ & $1(4.8)$ & 0.280 & 1.000 \\
\hline No & $\mathrm{n}=139$ & $93(93.9)$ & $26(86.7)$ & $20(95.2)$ & & \\
\hline Total number of chemotherapy courses & & & & & $<5$ & $\geq 5$ \\
\hline 1 & $\mathrm{n}=3$ & $2(2.0)$ & $1(3.3)$ & $0(0.0)$ & 0.001 & 0.157 \\
\hline 2 & $\mathrm{n}=4$ & $1(1.0)$ & $3(10.0)$ & $0(0.0)$ & & \\
\hline 3 & $\mathrm{n}=9$ & $4(4.0)$ & $4(13.3)$ & $1(4.8)$ & & \\
\hline 4 & $\mathrm{n}=29$ & $14(14.1)$ & $8(26.7)$ & $7(33.3)$ & & \\
\hline 5 & $\mathrm{n}=81$ & $59(59.6)$ & $11(36.7)$ & $11(52.4)$ & & \\
\hline 6 & $\mathrm{n}=24$ & $19(19.2)$ & $3(10.0)$ & $2(9.5)$ & & \\
\hline Chemotherapy dose reduction & & & & & Yes & No \\
\hline Yes & $\mathrm{n}=20$ & $15(15.2)$ & $3(10.0)$ & $2(9.5)$ & 0.564 & 0.734 \\
\hline No & $\mathrm{n}=130$ & $84(84.8)$ & $27(90.0)$ & $19(90.5)$ & & \\
\hline Ordinal number of first dose-reduced cour & & & & & & \\
\hline 3 & $\mathrm{n}=1$ & $0(0.0)$ & $1(33.3)$ & $0(0.0)$ & & \\
\hline 4 & $\mathrm{n}=5$ & $3(20.0)$ & $0(0.0)$ & $2(100.0)$ & & \\
\hline 5 & $\mathrm{n}=11$ & $9(60.0)$ & $2(66.7)$ & $0(0.0)$ & & \\
\hline 6 & $n=3$ & $3(20.0)$ & $0(0.0)$ & $0(0.0)$ & & \\
\hline Achieved portion of the target total dose & & & & & & \\
\hline $20-39 \%$ & $\mathrm{n}=3$ & $2(2.0)$ & $1(3.3)$ & $0(0.0)$ & & \\
\hline $40-59 \%$ & $\mathrm{n}=5$ & $1(1.0)$ & $4(13.3)$ & $0(0.0)$ & & \\
\hline $60-79 \%$ & $\mathrm{n}=12$ & $7(7.1)$ & $3(10.0)$ & $2(9.5)$ & & \\
\hline $80-99 \%$ & $\mathrm{n}=36$ & $20(20.2)$ & $10(33.3)$ & $6(28.6)$ & & \\
\hline $100-119 \%$ & $\mathrm{n}=74$ & $53(53.5)$ & $9(30.0)$ & $12(57.1)$ & & \\
\hline $120 \%$ & $\mathrm{n}=20$ & $16(16.2)$ & $3(10.0)$ & $1(4.8)$ & & \\
\hline Completion or exceeding & & & & & & \\
\hline of the target total dose & & & & & Yes & No \\
\hline Yes & $\mathrm{n}=94$ & $69(69.7)$ & $12(40.0)$ & $13(61.9)$ & 0.005 & 0.606 \\
\hline No & $\mathrm{n}=56$ & $30(30.3)$ & $18(60.0)$ & $8(38.1)$ & & \\
\hline CTCAE grading for increased creatinine & & & & & Grade $0 v$ & Grade $1 / 2$ \\
\hline Grade 0 & $\mathrm{n}=124$ & $88(88.9)$ & $24(80.0)$ & $12(57.1)$ & 0.225 & 0.001 \\
\hline Grade 1 & $\mathrm{n}=21$ & $9(9.1)$ & $5(16.7)$ & $7(33.3)$ & & \\
\hline Grade 2 & $\mathrm{n}=5$ & $2(2.0)$ & $1(3.3)$ & $2(9.5)$ & & \\
\hline CTCAE haematotoxicity grading & & & & & Grade $0 / 1 / 2$ & . Grade 3/4 \\
\hline Grade 0 & $\mathrm{n}=28$ & $24(24.2)$ & $3(10.0)$ & $1(4.8)$ & 0.004 & 0.083 \\
\hline Grade $1 / 2$ & $\mathrm{n}=81$ & $56(56.6)$ & $13(43.3)$ & $12(57.1)$ & & \\
\hline Grade $3 / 4$ & $\mathrm{n}=41$ & $19(19.2)$ & $14(46.7)$ & $8(38.1)$ & & \\
\hline Cause of haematotoxicity grade $3 / 4$ & & & & & & \\
\hline Leukocytes (Leuc) & $\mathrm{n}=22$ & $10(52.6)$ & $8(57.1)$ & $4(50.0)$ & & \\
\hline Haemoglobin $(\mathrm{Hb})$ & $\mathrm{n}=8$ & $3(15.8)$ & $4(28.6)$ & $1(12.5)$ & & \\
\hline Thrombocytes (Throm) & $\mathrm{n}=0$ & $0(0.0)$ & $0(0.0)$ & $0(0.0)$ & & \\
\hline Combination of Leuc, Hb, Throm & $\mathrm{n}=11$ & $6(31.6)$ & $2(14.3)$ & $3(37.5)$ & & \\
\hline
\end{tabular}

SIB: Simultaneous integrated boost; RTT: radiation treatment time; CTCAE: Common Terminology Criteria for Adverse Events. Bold $p$-Values indicate statistical significance. 
Table III. (Significant) Influencing factors that led to a change from cisplatin to carboplatin/paclitaxel evaluated in univariate and multivariate logistic regression.

\begin{tabular}{|c|c|c|c|c|c|c|}
\hline \multirow[t]{2}{*}{ Categories } & \multicolumn{2}{|c|}{ Regimen change } & \multirow{2}{*}{$\begin{array}{c}\text { Exponent (B) } \\
\text { Odds Ratio }\end{array}$} & \multicolumn{2}{|c|}{ Confidence interval $95 \%$} & \multirow[t]{2}{*}{$p$-Valuc } \\
\hline & $\begin{array}{c}\text { Yes } \\
n=21\end{array}$ & $\begin{array}{c}\text { No } \\
\mathrm{n}=99\end{array}$ & & $\begin{array}{l}\text { Lowest } \\
\text { Value }\end{array}$ & $\begin{array}{l}\text { Highest } \\
\text { Value }\end{array}$ & \\
\hline \multicolumn{7}{|c|}{ Univariate logistic regression } \\
\hline \multicolumn{7}{|l|}{ Age } \\
\hline \multicolumn{7}{|l|}{ Gender } \\
\hline Female $v s$. Male & & & 0.627 & 0.194 & 2.033 & 0.437 \\
\hline \multicolumn{7}{|l|}{ UICC } \\
\hline Stage III $v s$. Stage IV & & & 4.089 & 0.512 & 32.663 & 0.184 \\
\hline \multicolumn{7}{|l|}{ Tumour category } \\
\hline T 0/1/2 vs. T 3/4 & & & 1.875 & 0.671 & 5.241 & 0.231 \\
\hline \multicolumn{7}{|l|}{ Lymph nodes category } \\
\hline $\mathrm{N} 0 / 1$ vs. $\mathrm{N} 2 / 3$ & & & 1.625 & 0.547 & 4.823 & 0.382 \\
\hline Multilevel tumour & $17.5 \%$ & $82.5 \%$ & & & & \\
\hline Yes $v s$. No & & & 1.135 & 0.339 & 3.798 & 0.837 \\
\hline \multicolumn{7}{|l|}{$\mathrm{RCT}$ of a recurrence } \\
\hline Yes vs. No & & & 0.225 & 0.028 & 1.787 & 0.158 \\
\hline \multicolumn{7}{|l|}{ ACCI } \\
\hline$<4 v s . \geq 4$ & & & 4.165 & 1.308 & 13.263 & 0.016 \\
\hline \multicolumn{7}{|l|}{ Therapy } \\
\hline Definitive $v s$. adjuvant & & & 0.295 & 0.106 & 0.823 & 0.020 \\
\hline \multicolumn{7}{|l|}{ Nephrotoxicity } \\
\hline G 0 vs. G $1 / 2$ & & & 6.000 & 2.063 & 17.451 & 0.001 \\
\hline \multicolumn{7}{|c|}{ Multivariate logistic regression } \\
\hline \multicolumn{7}{|l|}{ Age } \\
\hline$\leq 60 \mathrm{vs} .>60$ years & & & 3.886 & 1.230 & 12.276 & 0.021 \\
\hline \multicolumn{7}{|l|}{ Therapy } \\
\hline Definitive $v s$. adjuvant & $17.5 \%$ & $82.5 \%$ & 0.330 & 0.109 & 0.997 & 0.049 \\
\hline \multicolumn{7}{|l|}{ Nephrotoxicity } \\
\hline G $0 v s . \mathrm{G} 1 / 2$ & & & 3.574 & 1.085 & 11.775 & 0.036 \\
\hline
\end{tabular}

UICC: Union Internationale Contre le Cancer; RCT: radiochemotherapy; ACCI: Age-Adjusted Charlson Comorbidity Index. Bold $p$-Values indicate statistical significance.

Table IV. p-Values based on Fisher's exact tests comparing toxicity and outcome parameters in each group versus the two other groups.

\begin{tabular}{llccc}
\hline Categories & & $\begin{array}{c}\text { Cisplatin } \\
\text { Group 1 }\end{array}$ & $\begin{array}{c}\text { Carboplatin/Paclitaxel } \\
\text { Group 2 }\end{array}$ & $\begin{array}{c}\text { Regimen change } \\
\text { Group 3 }\end{array}$ \\
\hline Nephrotoxicity & G 0 vs. G 1/2 & $\mathbf{0 . 0 1 1}$ & 0.787 & 0.003 \\
Haematotoxicity & G 0 vs. G 1-4 & $\mathbf{0 . 0 1 5}$ & 0.202 & 0.127 \\
Haematotoxicity & G 0/1/2 vs. G 3/4 & $\mathbf{0 . 0 0 3}$ & $\mathbf{0 . 0 1 2}$ & 0.291 \\
RTT $>$ 49 days & Yes vs. No & 0.511 & 0.231 & 1.000 \\
Recurrence after RCT & Yes vs. No & 0.302 & 0.529 & 1.000 \\
Metastases after RCT & Yes vs. No & 0.803 & 1.000 & 0.741 \\
\hline
\end{tabular}

RTT: Radiation treatment time; RCT: radiochemotherapy. Bold $p$-Values indicate statistical significance.

changing between both regimens in definitive or adjuvant RCTs in locally advanced squamous cell head and neck cancer and evaluated toxicity and outcome parameters. The results demonstrated that cisplatin application led to CTx continuation and achievement of the target number of 5 courses more frequently than carboplatin/paclitaxel. Group 2 patients exhibited the most severe comorbidities; therefore, carboplatin/paclitaxel was administered from the start of therapy. In addition, haematotoxicity was highest in patients receiving carboplatin/paclitaxel, and renal toxicity 


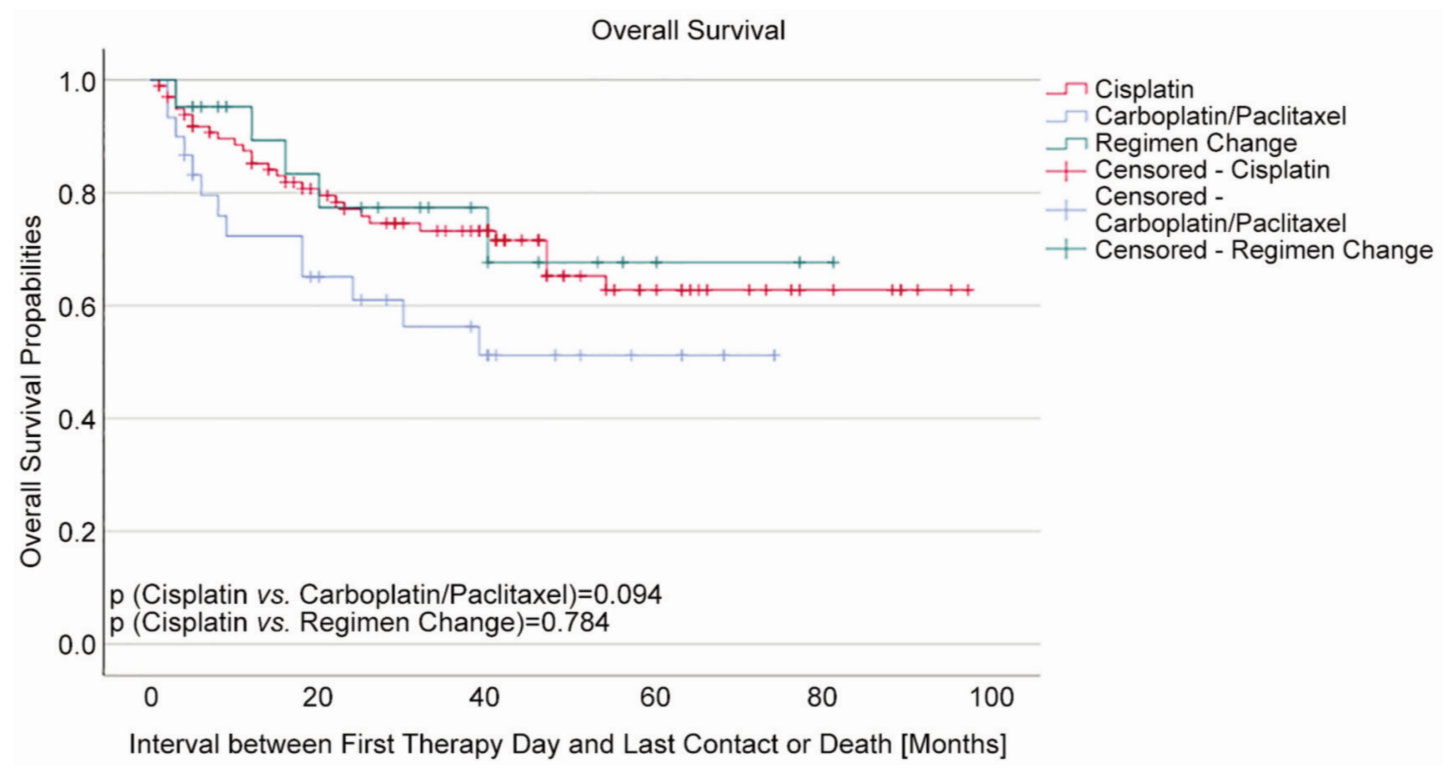

Figure 1. Kaplan-Meier curves of overall survival for comparison between the cisplatin, carboplatin/paclitaxel, and regimen change group.

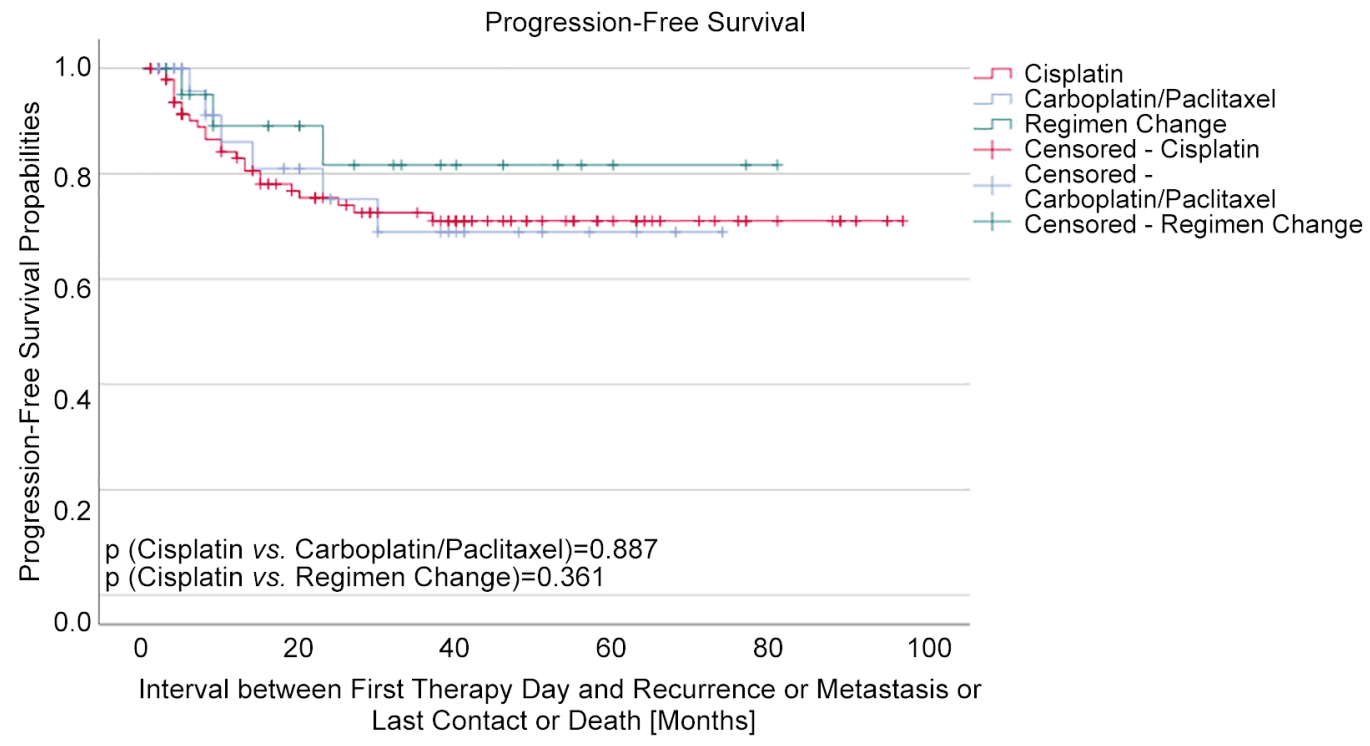

Figure 2. Kaplan-Meier curves of progression-free survival for comparison between the cisplatin, carboplatin/paclitaxel, and regimen change group.

was greatest in the Change Group, explaining the change in the cytostatic drug from cisplatin to carboplatin/paclitaxel during therapy. However, none of the three groups differed significantly in OS, PFS, or LRC, which may be attributable to our study's limiting factors, including its retrospective character and small group sizes. To date, few studies have compared the toxicity and outcome of CRT with cisplatin $v s$. carboplatin-based regimens in locally advanced head and neck tumour patients. To our knowledge, this is the first study to show data from patients who switched from cisplatin to carboplatin/paclitaxel during the course of therapy.

Noronha et al. presented reasons for not using cisplatin, such as low creatinine clearance in $65.07 \%$ of cases, sensorineural hearing loss in $28.57 \%$, uncontrolled comorbidities in $4.76 \%$ and advanced age in $1.60 \%$ of 


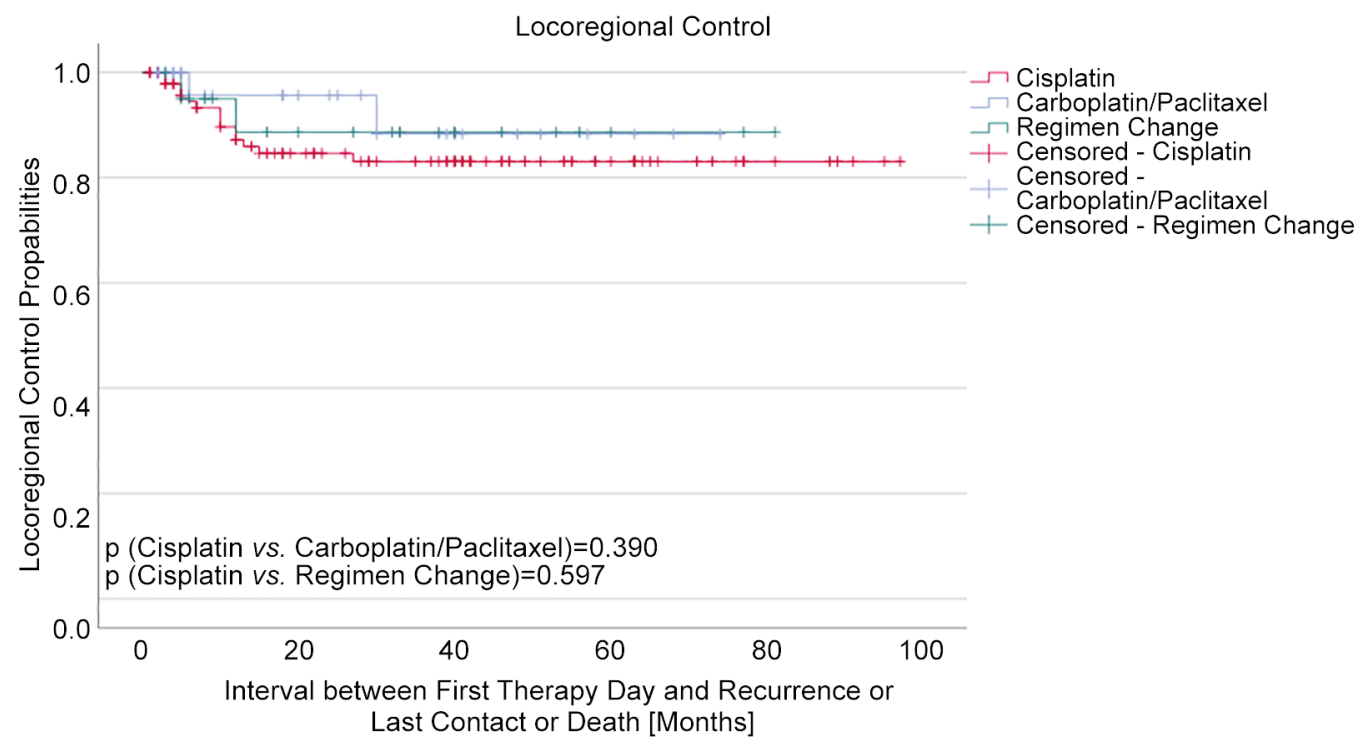

Figure 3. Kaplan-Meier curves of locoregional control for comparison between the cisplatin, carboplatin/paclitaxel, and regimen change group.

patients. Sixteen of the 63 patients suffered from one or more of the following comorbidities: diabetes, hypertension, past cerebrovascular accident (CVA), asthma, and hepatitis B virus surface antigen (HBsAg) positivity (10). In contrast to our study, the work by Noronha et al. did not apply CCI to measure comorbidities, making it difficult to define which patients had more comorbidities. However, the reasons for not utilizing cisplatin confirm what we considered relevant.

Although not concerning head and neck cancer, a study by Birtle et al. evaluating the efficacy of systemic platinumbased CTx in patients with upper urinary tract urothelial carcinomas after nephroureterectomy with curative intent in a phase III trial caught our attention. In that study, 6 out of 76 patients changed from planned therapy with gemcitabinecisplatin to gemcitabine-carboplatin before treatment began, and another 10 patients switched in the second cycle or later: 6 of these cases could be associated with a reduction in glomerular filtration rate (GFR), 2 with suspected renal failure, and 2 more with grade 3 toxicity manifested as joint pain or tinnitus (29). In the study of Chitapanarux et al., leucocytopaenia as well as anaemia and renal toxicity were predominant in the cisplatin group, while thrombocytopaenia was predominant in the carboplatin group (9). In our cohort, both anaemia and leucocytopaenia alone were more prevalent in patients receiving carboplatin/paclitaxel than in those receiving cisplatin. Thrombocytopaenia, which always appeared in combination with anaemia and/or leucocytopaenia, likewise occurred more frequently in the cisplatin group. Of note, both our analyses and those of Chitapanarux et al. indicated an association between cisplatin and renal toxicity that was absent in the carboplatin/paclitaxel arm. Rades et al. also compared cisplatin and carboplatin monotherapy in adjuvant RCTs for locally advanced tumours of the oropharynx and oral cavity. While both regimens led to acute toxicities, including mucositis, nausea/vomiting, haematotoxicity, renal toxicity and skin toxicity, as well as late toxicities, such as xerostomia, neck fibrosis, lymphoedema and skin toxicity, differences between the cisplatin and carboplatin groups were not significant (30). In contrast, our findings reveal a significant association of renal toxicity of any degree with the cisplatin cohort and haematotoxicity with both the cisplatin and carboplatin/paclitaxel cohorts. Based on practical experience in our department, we advocate the use of carboplatin alone when paclitaxel administration is not possible, for example, in case of allergy. However, we do not have our own study results in this regard.

Regarding survival, a randomized study by Homma et al. involving 119 patients with squamous cell carcinoma of the head and neck who received weekly carboplatin $100 \mathrm{mg} / \mathrm{m}^{2}$ or daily low-dose cisplatin $4 \mathrm{mg} / \mathrm{m}^{2}$ each during the first 4 weeks of RT offers some insights. Their conclusion of favouring weekly carboplatin in the frame of concomitant RCT contrasts with those of our study supporting cisplatin as the preferable choice for adjuvant therapy. However, Homma et al. noted that the total applied cisplatin dose of $64 \mathrm{mg} / \mathrm{m}^{2}$ may have been too low to be effective. They reported 5-year rates for LRC of $56.2 \%$ in the carboplatin group and $35.5 \%$ in the cisplatin group $(p<0.05)$, with no significant difference in 5-year OS rates, which were $71.4 \%$ in the carboplatin group and $66.0 \%$ in the cisplatin arm (31). In their work comparing cisplatin and carboplatin in adjuvant RCT for locally advanced tumours of 
the oropharynx and oral cavity, Rades et al. also determined the 3-year rates for outcome parameters: LRC rates were $85.0 \%$ in the cisplatin and $62.0 \%$ in the carboplatin cohort ( $p=0.004)$, while those of OS reached $78.0 \%$ in the cisplatin and $51.0 \%$ in the carboplatin group ( $p=0.001)$ (30). Comparing their results with ours, neither LRC nor OS showed any significant differences between the two groups. Of note, the application of the cytostatic drug was not weekly as in our study, but rather consisted of two courses of cisplatin $20 \mathrm{mg} / \mathrm{m}^{2}$ on days 1-5 and 29-33 or two courses of carboplatin AUC 1.5 on days 1-5 and 29-33 (30). In this context, it is worth considering another study by Rades et al. on definitive radiochemotherapy of locally advanced head and neck cancer, showing that weekly cisplatin $30-40 \mathrm{mg} / \mathrm{m}^{2}$ was inferior to 3 courses of cisplatin $100 \mathrm{~m} / \mathrm{m}^{2}$ on days 1,22 , and 43 in terms of OS and LRC (32). Yet a further recent 2019 study by Helfenstein et al. found that significantly more head and neck tumour patients achieved a cumulative cisplatin dose of $\geq 200$ $\mathrm{mg} / \mathrm{m}^{2}$ in the setting of an RCT when treated with a 3-week regimen compared with weekly dosing. However, a cumulative cisplatin dose $\geq 200 \mathrm{mg} / \mathrm{m}^{2}$ was not associated with improved outcome in this study (33). In a nasopharyngeal carcinoma study by Chitapanarux et al., the 3-year OS rates were $77.7 \%$ in the cisplatin arm and $79.2 \%$ in the carboplatin arm ( $p=0.9884)$ (9). As in our study, no significance was found $(p=0.094)$. However, another team set out to assess outcome parameters in patients with locally advanced nasopharyngeal carcinoma: Luttke et al. matched a standard group of 238 patients receiving standard RCT and an intervention group of 242 patients undergoing 3 cycles of induction CTx with gemcitabine and cisplatin followed by concomitant RCT with cisplatin in the IMRT technique. The result was a significantly better relapse-free survival at 3 years of $85.3 \%$ in the intervention arm compared with $76.5 \%$ in the standard arm. Moreover, improved 3-year OS was observed in the induction CTx group, with $94.6 \%$ vs. $90.3 \%$ in the standard group. However, the intervention group faced comparatively higher toxicities and side effects, mainly neutropaenia, thrombocytopaenia, anaemia, nausea and vomiting (34). Regarding paclitaxel as an alternative for patients who cannot tolerate standard platinum-based therapies, a recent study by Okada et al. is of great value, evaluating the safety and efficacy of the combination of paclitaxel and cetuximab in patients with recurrent/metastatic squamous cell carcinoma of the head and neck (R/M SCCHN). Overall response and disease control rates of $43 \%$ and $79 \%$, respectively, were observed with firstline therapy, while rates of $20 \%$ and $90 \%$, respectively, were achieved with second-line and later therapies. With a median PFS of 5.3 months and a median OS of 12.5 months, it was concluded that the aforementioned combination therapy may be appropriate. Adverse events such as neutropenia (grade 3/4) and anemia, which affected $8-13 \%$ of the patients studied, were all considered manageable (35). Another study by Sato et al. was devoted to evaluating the efficacy of paclitaxel-based CTx in patients with head and neck cancer. They studied 12 patients who received CTx with paclitaxel before and 10 patients after nivolumab. Thereby it was found that paclitaxel-based CTx had a significantly better overall response rate (ORR) of $70 \%$ ( $p=0.027)$ and time to progression (TTP) of 7.4 months $(p=0.020)$ after nivolumab compared with an ORR of $17 \%$ and TTP of 4.9 months before nivolumab (36). Regardless of whether cisplatin or carboplatin/paclitaxel was applied, one would expect the achievement of the targeted 5 courses to have a positive effect on survival. In contrast, we found, as did Steinmann et al. (5), that the number of courses was not critical. Nonetheless, other studies have found that a cumulative cisplatin dose $\geq 200 \mathrm{mg} / \mathrm{m}^{2}$ resulted in a significantly better outcome than a cumulative dose of $<200$ $\mathrm{mg} / \mathrm{m}^{2}(3,4)$. A randomized trial by Chitapanarux et al. evaluated RCTs with cisplatin vs. carboplatin in the management of locally advanced nasopharyngeal carcinoma, treating 101 patients with cisplatin and 105 with carboplatin in the case of adjuvant therapy combined with 5-fluorouracil. The results showed planned RCT completion in $59.0 \%$ of cases in the cisplatin group compared with $73.0 \%$ in the carboplatin group (9). In contrast to these findings, our study revealed that $69.7 \%$ of cisplatin and only $40.0 \%$ of carboplatin/paclitaxel patients finished the scheduled 5 CTx courses. A further trial by Dahlke et al. on treatment-related factors, such as total treatment time and radiotherapy time (RTT) in head and neck cancer patients, reported a median RTT of 40 days (37) - in our cohort, the median was 43 days, with 42 days in the cisplatin cohort and 43 days each in the carboplatin/paclitaxel and regimen change group. RT length (RT $\leq 49$ days vs. RT $>49$ days) was not significant in the univariate logistic regression examining factors affecting the cytostatic drug transition from cisplatin to carboplatin/paclitaxel. Likewise, no dependency was found between RT duration $>49$ days and the three groups tested. In another trial, Chiang et al. investigated the prognostic impact of missed RT sessions in patients undergoing surgery for oral squamous cell carcinoma. As a result, they found 5year OS to be $53.0 \%, 58.1 \%$ and $64.5 \%$ in the early missed RT, late missed RT and RT-as-planned groups, respectively $(p=0.046)$. Early missed RT was independently associated with both poorer OS $(p=0.006)$ and the occurrence of distant metastases $(p=0.031)(38)$.

Speaking about the management of cancer in the elderly, there are different perspectives as to what is the most appropriate approach. For head and neck cancer patients, both radiotherapy alone (39) and combined radiochemotherapy (40) are feasible strategies. When it comes to esophageal cancer, radiochemotherapy in older patients resulted in outcomes comparable to those of younger patients (41-43). In elderly patients with non-small-cell lung carcinoma (NSCLC), combined radiochemotherapy even resulted in improved survival compared with radiotherapy alone $(44,45)$. 
On all accounts, the indication for irradiation of elderly tumour patients should be made in dependence of general condition and comorbidities; age alone rarely is considered a contraindication for radiotherapy. Thus, also dose reduction, especially with regard to a curative approach, does not seem to be justified solely by reason of age (46).

Limitations. One of the limiting factors is our study's retrospective nature, which may bias our results, particularly in the context of selection bias. An additional constraint could be the follow-up time, which, with a median of 41 months, may still be too short to draw reliable conclusions on survival data. Furthermore, our analysed collective partly consists of small group sizes, especially regarding group 3 .

However, another limitation we encountered was the CCI ignoring psychological parameters and diseases, making a holistic evaluation of comorbidities difficult. Nevertheless, the CCI served its most crucial purpose in our study, namely, to make comorbidities measurable and comparable in the three groups. The uneven distribution of age in the groups can also be listed within the limitations. However, the presence of elderly patients in groups 2 and 3 supports the fact that, according to our findings, an age of more than 60 years is significantly associated with a change of cytostatic drug from cisplatin to carboplatin/paclitaxel. In addition, HPV/p16 status was not yet regularly determined in the laboratory during the treatment period of our collective's individuals, and therefore could not be considered in patients with oropharyngeal carcinoma.

\section{Conclusion}

Although potentially leading to higher nephrotoxicity levels, cisplatin-oriented combined RCT remains the recommended treatment for definitive and adjuvant RCT of patients with locally advanced head and neck cancer. Even when combined with a switch to carboplatin/paclitaxel during the course of therapy, the use of cisplatin proves to be the superior choice by achieving 5 targeted CTx courses. In summary, therapy initiation with cisplatin is advised if the patient's general condition allows. Patients who change from cisplatin to carboplatin/paclitaxel do not suffer any survival disadvantage in comparison. However, treatment with carboplatin/paclitaxel offers a valid alternative for patients unfit for cisplatin, despite its association with haematotoxicity and a higher likelihood of noncompletion of planned CTx. Because of their retrospective nature, the results should be considered critically and confirmed in a prospective study.

\section{Conflicts of Interest}

The Authors declare that they have no competing interests regarding this study.

\section{Authors' Contributions}

All Authors contributed to the study conception and design. JW, SV, and $\mathrm{HC}$ were significantly involved in writing and revising the manuscript, while DoS played an essential role in the statistical analyses and their interpretation. DS supervised our work from the beginning and made seminal decisions in consultation with the team. All Authors read and approved the final manuscript.

\section{References}

1 Pignon JP, le Maître A, Bourhis J and MACH-NC Collaborative Group: Meta-analyses of chemotherapy in head and neck cancer (MACH-NC): an update. Int J Radiat Oncol Biol Phys 69(2 Suppl): S112-S114, 2007. PMID: 17848275. DOI: 10.1016/ j.ijrobp.2007.04.088

2 Guan J, Li Q, Zhang Y, Xiao N, Chen M, Zhang Y, Li L and Chen L: A meta-analysis comparing cisplatin-based to carboplatin-based chemotherapy in moderate to advanced squamous cell carcinoma of head and neck (SCCHN). Oncotarget 7(6): 7110-7119, 2016. PMID: 26755647. DOI: 10.18632 /oncotarget.6858

3 Spreafico A, Huang SH, Xu W, Granata R, Liu CS, Waldron JN, Chen E, Ringash J, Bayley A, Chan KK, Hope AJ, Cho J, Razak AA, Hansen A, Jang R, Perez-Ordonez B, Weinreb I, Bossi P, Orlandi E, Licitra LF, Song Y, O'Sullivan B, Siu LL and Kim J: Impact of cisplatin dose intensity on human papillomavirusrelated and -unrelated locally advanced head and neck squamous cell carcinoma. Eur J Cancer 67: 174-182, 2016. PMID: 27669504. DOI: 10.1016/j.ejca.2016.08.013

4 Al-Mamgani A, de Ridder M, Navran A, Klop WM, de Boer JP and Tesselaar ME: The impact of cumulative dose of cisplatin on outcome of patients with head and neck squamous cell carcinoma. Eur Arch Otorhinolaryngol 274(10): 3757-3765, 2017. PMID: 28755023. DOI: 10.1007/s00405-017-4687-4

5 Steinmann D, Cerny B, Karstens JH and Bremer M: Chemoradiotherapy with weekly cisplatin $40 \mathrm{mg} / \mathrm{m}(2)$ in 103 head-and-neck cancer patients: a cumulative dose-effect analysis. Strahlenther Onkol 185(10): 682-688, 2009. PMID: 19806334. DOI: $10.1007 / \mathrm{s} 00066-009-1989-5$

6 Adelstein DJ, Li Y, Adams GL, Wagner H Jr, Kish JA, Ensley JF, Schuller DE and Forastiere AA: An intergroup phase III comparison of standard radiation therapy and two schedules of concurrent chemoradiotherapy in patients with unresectable squamous cell head and neck cancer. J Clin Oncol 21(1): 92-98, 2003. PMID: 12506176. DOI: 10.1200/JCO.2003.01.008

7 Dugbartey GJ, Peppone LJ and de Graaf IA: An integrative view of cisplatin-induced renal and cardiac toxicities: Molecular mechanisms, current treatment challenges and potential protective measures. Toxicology 371: 58-66, 2016. PMID: 27717837. DOI: $10.1016 /$ j.tox.2016.10.001

8 Corry J, Bressel M, Fua T, Herschtal A, Solomon B, Porceddu SV, Wratten C and Rischin D: Prospective study of cetuximab, carboplatin, and radiation therapy for patients with locally advanced head and neck squamous cell cancer unfit for cisplatin. Int J Radiat Oncol Biol Phys 98(4): 948-954, 2017. PMID: 28483336. DOI: 10.1016/j.ijrobp.2017.02.088

9 Chitapanarux I, Lorvidhaya V, Kamnerdsupaphon P, Sumitsawan Y, Tharavichitkul E, Sukthomya V and Ford J: Chemoradiation comparing cisplatin versus carboplatin in locally advanced 
nasopharyngeal cancer: randomised, non-inferiority, open trial. Eur J Cancer 43(9): 1399-1406, 2007. PMID: 17467265. DOI: 10.1016/j.ejca.2007.03.022

10 Noronha V, Sharma V, Joshi A, Patil VM, Laskar SG and Prabhash K: Carboplatin-based concurrent chemoradiation therapy in locally advanced head and neck cancer patients who are unfit for cisplatin therapy. Indian J Cancer 54(2): 453-457, 2017. PMID: 29469077. DOI: 10.4103/ijc.IJC_320_17

11 Maring S, Elsayad K, Stenner M, Rudack C, Haverkamp U, Rehkämper J, Wardelmann E and Eich HT: Efficacy of carboplatin/paclitaxel-based radiochemotherapy in locally advanced squamous cell carcinoma of head and neck. Oncol Res Treat 41(12): 736-743, 2018. PMID: 30419553. DOI: 10.1159/ 000494031

12 Agarwala SS, Cano E, Heron DE, Johnson J, Myers E, Sandulache V, Bahri S, Ferris R, Wang Y and Argiris A: Longterm outcomes with concurrent carboplatin, paclitaxel and radiation therapy for locally advanced, inoperable head and neck cancer. Ann Oncol 18(7): 1224-1229, 2007. PMID: 17675395. DOI: $10.1093 /$ annonc/mdm088

13 Suntharalingam M, Haas ML, Conley BA, Egorin MJ, Levy S, Sivasailam S, Herman JM, Jacobs MC, Gray WC, Ord RA, Aisner JA and Van Echo DA: The use of carboplatin and paclitaxel with daily radiotherapy in patients with locally advanced squamous cell carcinomas of the head and neck. Int J Radiat Oncol Biol Phys 47(1): 49-56, 2000. PMID: 10758304. DOI: 10.1016/s0360-3016(00)00408-9

14 van Hagen P, Hulshof MC, van Lanschot JJ, Steyerberg EW, van Berge Henegouwen MI, Wijnhoven BP, Richel DJ, Nieuwenhuijzen GA, Hospers GA, Bonenkamp JJ, Cuesta MA, Blaisse RJ, Busch OR, ten Kate FJ, Creemers GJ, Punt CJ, Plukker JT, Verheul HM, Spillenaar Bilgen EJ, van Dekken H, van der Sangen MJ, Rozema T, Biermann K, Beukema JC, Piet AH, van Rij CM, Reinders JG, Tilanus HW, van der Gaast A and CROSS Group: Preoperative chemoradiotherapy for esophageal or junctional cancer. N Engl J Med 366(22): 2074-2084, 2012. PMID: 22646630. DOI: 10.1056/NEJMoa1112088

15 Honing J, Smit JK, Muijs CT, Burgerhof JGM, de Groot JW, Paardekooper G, Muller K, Woutersen D, Legdeur MJC, Fiets WE, Slot A, Beukema JC, Plukker JTM and Hospers GAP: A comparison of carboplatin and paclitaxel with cisplatinum and 5-fluorouracil in definitive chemoradiation in esophageal cancer patients. Ann Oncol 25(3): 638-643, 2014. PMID: 24492674. DOI: $10.1093 /$ annonc/mdt589

16 Langer CJ, Leighton JC, Comis RL, O’Dwyer PJ, McAleer CA, Bonjo CA, Engstrom PF, Litwin S and Ozols RF: Paclitaxel and carboplatin in combination in the treatment of advanced nonsmall-cell lung cancer: a phase II toxicity, response, and survival analysis. J Clin Oncol 13(8): 1860-1870, 1995. PMID: 7543559. DOI: $10.1200 / J C O .1995 .13 .8 .1860$

17 Charlson ME, Pompei P, Ales KL and MacKenzie CR: A new method of classifying prognostic comorbidity in longitudinal studies: development and validation. J Chronic Dis 40(5): 373-383, 1987. PMID: 3558716. DOI: 10.1016/0021-9681(87)90171-8

18 Roffman CE, Buchanan J and Allison GT: Charlson Comorbidities Index. J Physiother 62(3): 171, 2016. PMID: 27298055. DOI: 10.1016/j.jphys.2016.05.008

19 Singh B, Bhaya M, Stern J, Roland JT, Zimbler M, Rosenfeld RM, Har-El G and Lucente FE: Validation of the Charlson comorbidity index in patients with head and neck cancer: a multi-institutional study. Laryngoscope 107(11 Pt 1): 1469-1475, 1997. PMID: 9369392. DOI: 10.1097/00005537-19971100000009

20 Austin SR, Wong YN, Uzzo RG, Beck JR and Egleston BL: Why summary comorbidity measures such as the Charlson Comorbidity Index and Elixhauser score work. Med Care 53(9): e65-e72, 2015. PMID: 23703645. DOI: 10.1097/MLR.0b013e318297429c

21 Charlson M, Szatrowski TP, Peterson J and Gold J: Validation of a combined comorbidity index. J Clin Epidemiol 47(11): 1245-1251, 1994. PMID: 7722560. DOI: 10.1016/0895-4356(94) 90129-5

22 Yang CC, Chen PC, Hsu CW, Chang SL and Lee CC: Validity of the age-adjusted Charlson comorbidity index on clinical outcomes for patients with nasopharyngeal cancer post radiation treatment: a 5-year nationwide cohort study. PLoS One 10(1): e0117323, 2015. PMID: 25617629. DOI: 10.1371/journal.pone.0117323

23 Hall WH, Ramachandran R, Narayan S, Jani AB and Vijayakumar S: An electronic application for rapidly calculating Charlson comorbidity score. BMC Cancer 4: 94, 2004. PMID: 15610554. DOI: 10.1186/1471-2407-4-94

24 Klüter S, Katayama S, Spindeldreier CK, Koerber SA, Major G, Alber M, Akbaba S, Debus J and Hörner-Rieber J: First prospective clinical evaluation of feasibility and patient acceptance of magnetic resonance-guided radiotherapy in Germany. Strahlenther Onkol 196(8): 691-698, 2020. PMID: 32002567. DOI: $10.1007 / \mathrm{s} 00066-020-01578-\mathrm{z}$

25 Welz S and Zips D: [FDG-PET/CT for exclusion of cervical lymph node metastases in clinically N0 head and neck tumors]. Strahlenther Onkol 196(2): 200-201, 2020. PMID: 31938807. DOI: $10.1007 / \mathrm{s} 00066-019-01569-9$

26 Shah J and Montero P: New AJCC/UICC staging system for head and neck, and thyroid cancer. Revista Médica Clínica Las Condes 29(4): 397-404, 2018. DOI: 10.1016/j.rmclc.2018.07.002

27 Zhang S, Chen Q and Wang Q: The use of and adherence to CTCAE v3.0 in cancer clinical trial publications. Oncotarget 7(40): 65577-65588, 2016. PMID: 27564109. DOI: 10.18632/ oncotarget. 11576

28 Katz MH: Study design and statistical analysis: A practical guide for clinicians. Cambridge, Cambridge University Press, 2006.

29 Birtle A, Johnson M, Chester J, Jones R, Dolling D, Bryan RT, Harris C, Winterbottom A, Blacker A, Catto JWF, Chakraborti P, Donovan JL, Elliott PA, French A, Jagdev S, Jenkins B, Keeley FX Jr, Kockelbergh R, Powles T, Wagstaff J, Wilson C, Todd R, Lewis R and Hall E: Adjuvant chemotherapy in upper tract urothelial carcinoma (the POUT trial): a phase 3, open-label, randomised controlled trial. Lancet 395(10232): 1268-1277, 2020. PMID: 32145825. DOI: 10.1016/S01406736(20)30415-3

30 Rades D, Ulbricht T, Hakim SG and Schild SE: Cisplatin superior to carboplatin in adjuvant radiochemotherapy for locally advanced cancers of the oropharynx and oral cavity. Strahlenther Onkol 188(1): 42-48, 2012. PMID: 22194029. DOI: 10.1007/ s00066-011-0005-z

31 Homma A, Shirato H, Furuta Y, Nishioka T, Oridate N, Tsuchiya $\mathrm{K}$, Nagahashi $\mathrm{T}$, Aoyama $\mathrm{H}$, Inuyama $\mathrm{Y}$ and Fukuda $\mathrm{S}$ : Randomized phase II trial of concomitant chemoradiotherapy using weekly carboplatin or daily low-dose cisplatin for squamous cell carcinoma of the head and neck. Cancer J 10(5): 326-332, 2004. PMID: 15530262. DOI: 10.1097/00130404200409000-00010 
32 Rades D, Seidl D, Janssen S, Bajrovic A, Karner K, Strojan P and Schild SE: Comparison of weekly administration of cisplatin versus three courses of cisplatin $100 \mathrm{mg} / \mathrm{m}(2)$ for definitive radiochemotherapy of locally advanced head-and-neck cancers. BMC Cancer 16: 437, 2016. PMID: 27391309. DOI: 10.1186/ s12885-016-2478-8

33 Helfenstein S, Riesterer O, Meier UR, Papachristofilou A, Kasenda B, Pless M and Rothschild SI: 3-weekly or weekly cisplatin concurrently with radiotherapy for patients with squamous cell carcinoma of the head and neck - a multicentre, retrospective analysis. Radiat Oncol 14(1): 32, 2019. PMID: 30744643. DOI: 10.1186/s13014-019-1235-y

34 Luttke M, Späth R, Marschner S and Walter F: [Induction chemotherapy with gemcitabine and cisplatin followed by radiochemotherapy in locally advanced nasopharyngeal carcinoma]. Strahlenther Onkol 196(8): 740-742, 2020. PMID: 32524161. DOI: $10.1007 / \mathrm{s} 00066-020-01644-6$

35 Dahlke S, Steinmann D, Christiansen H, Durisin M, Eckardt A, Wegener G, Bremer M and Meyer A: Impact of time factors on outcome in patients with head and neck cancer treated with definitive radio(chemo)therapy. In Vivo 31(5): 949-955, 2017. PMID: 28882964. DOI: 10.21873 /invivo.11152

36 Okada T, Okamoto I, Sato H, Ito T, Miyake K and Tsukahara K: Efficacy and safety of paclitaxel combined with cetuximab for head and neck squamous cell carcinoma. In Vivo 35(2): 12531259, 2021. PMID: 33622928. DOI: 10.21873/invivo.12376

37 Sato Y, Fukuda N, Fujiwara YU, Wang X, Urasaki T, Ohmoto A, Nakano K, Ono M, Tomomatsu J, Mitani H and Takahashi S: Efficacy of paclitaxel-based chemotherapy after progression on nivolumab for head and neck cancer. In Vivo 35(2): 1211-1215, 2021. PMID: 33622923. DOI: 10.21873/invivo.12371

38 Chiang YY, Chou YC, Chang KP, Liao CT, Wu YY, Yap WK, Pai PC, Chang JT, Lin CY, Fan KH, Huang BS, Hung TM and Tsang NM: Missed radiation therapy sessions in first three weeks predict distant metastasis and less favorable outcomes in surgically treated patients with oral cavity squamous cell carcinoma. Radiat Oncol 15(1): 194, 2020. PMID: 32795324. DOI: $10.1186 / \mathrm{s} 13014-020-01632-1$

39 Haehl E, Rühle A, David H, Kalckreuth T, Sprave T, Stoian R, Becker C, Knopf A, Grosu AL and Nicolay NH: Radiotherapy for geriatric head-and-neck cancer patients: what is the value of standard treatment in the elderly? Radiat Oncol 15(1): 31, 2020. PMID: 32019576. DOI: 10.1186/s13014-020-1481-z
40 Müller von der Grün J, Martin D, Stöver T, Ghanaati S, Rödel $\mathrm{C}$ and Balermpas P: Chemoradiotherapy as definitive treatment for elderly patients with head and neck cancer. Biomed Res Int 2018: 3508795, 2018. PMID: 29581971. DOI: 10.1155/2018/ 3508795

41 Rochigneux P, Resbeut M, Rousseau F, Bories E, Raoul JL, Poizat F and Moureau-Zabotto L: Radio(chemo)therapy in elderly patients with esophageal cancer: a feasible treatment with an outcome consistent with younger patients. Front Oncol 4: 100, 2014. PMID: 24860783. DOI: 10.3389/fonc.2014.00100

42 Münch S, Heinrich C, Habermehl D, Oechsner M, Combs SE and Duma MN: Primary radio(chemo)therapy for esophageal cancer in elderly patients: are efficiency and toxicity comparable with younger patients? Eur J Med Res 22(1): 24, 2017. PMID: 28683820. DOI: 10.1186/s40001-017-0265-X

43 Semrau R, Herzog SL, Vallböhmer D, Kocher M, Hölscher A and Müller RP: Radiotherapy in elderly patients with inoperable esophageal cancer. Is there a benefit? Strahlenther Onkol 188(3): 226-232, 2012. PMID: 22318327. DOI: 10.1007/s00066-0110039-2

44 Miller ED, Fisher JL, Haglund KE, Grecula JC, Xu-Welliver M, Bertino EM, He K, Shields PG, Carbone DP, Williams TM, Otterson GA and Bazan JG: The addition of chemotherapy to radiation therapy improves survival in elderly patients with stage III non-small cell lung cancer. J Thorac Oncol 13(3): 426-435, 2018. PMID: 29326090. DOI: 10.1016/j.jtho 2017.11 .135

45 Dawe DE, Christiansen D, Swaminath A, Ellis PM, Rothney J, Rabbani R, Abou-Setta AM, Zarychanski R and Mahmud SM: Chemoradiotherapy versus radiotherapy alone in elderly patients with stage III non-small cell lung cancer: A systematic review and meta-analysis. Lung Cancer 99: 180-185, 2016. PMID: 27565937. DOI: 10.1016/j.lungcan.2016.07.016

46 Geinitz H, Zimmermann FB and Molls M: [Radiotherapy of the elderly patient. Radiotherapy tolerance and results in older patients]. Strahlenther Onkol 175(3): 119-127, 1999. PMID: 10093614. DOI: $10.1007 / B F 02742345$
Received January 9, 2022

Revised February 8, 2022

Accepted February 9, 2022 